Western University

Scholarship@Western

Department of Economics Research Reports

Economics Working Papers Archive

1975

\title{
The Bias and Mean Squared Error of Forecasts from Partially Restricted Reduced Form
}

\author{
A. L. Nagar \\ S. N. Sahay
}

Follow this and additional works at: https://ir.lib.uwo.ca/economicsresrpt

Part of the Economics Commons

Citation of this paper:

Nagar, A. L., S. N. Sahay. "The Bias and Mean Squared Error of Forecasts from Partially Restricted Reduced Form." Department of Economics Research Reports, 7514. London, ON: Department of Economics, University of Western Ontario (1975). 
Research Report 7514

THE BIAS AND MEAN SQUARED ERROR OF FORECASTS FROM PARTIALLY RESTRICTED

REDUCED FORM

by
A. L. Nagar
and
S. N. Sahay

June 1975 
The Bias and Mean Squared Error of Forecasts

From Partially Restricted Reduced Form

by

\author{
A. L. Nagar and S. N. Sahay \\ The University of Western Ontario \\ and \\ The Delhi School of Economics
}

\title{
1. Introduction
}

An important function of a simultaneous equations model is to help in predicting the values of endogenous variables given the values of the exogenous variables. In fact prediction errors are good indicators of the performance of such a model in practice. Asymptotic variance-covariance matrix of forecasts has been worked out by Hooper and Zellner (1961) and Goldberger, Nagar and Odeh (1961). The approach adopted by Hooper and Zellner was that of obtaining forecasts from unrestricted reduced form and Goldberger, Nagar and Odeh obtain forecasts from restricted (or derived) reduced form. Relative merits of the two methods have been investigated by several authors including Mosbaek and Wold (1969). In this paper we shall investigate properties of forecasts obtained according to partially restricted reduced form. This procedure was originally proposed by Amemiya (1966) and later analyzed by Kakwani and Court (1972).

The specification of the linear structural model and the reduced form has been given in Section 2 and the method of partially restricted reduced form has been spelt out in Section 3. In section 4 we analyze the exact bias and mean squared error of forecast obtained according to the partially restricted reduced form when there are only two endogenous variables in the given structural equation. We also obtain the asymptotic approximations to the bias and mean squared error so obtained. Section 5 gives the proofs of the theorems. 


\section{Linear Structural System and its Reduced Form}

Let us write the complete equation system in M linear structural equations in $M$ endogenous and $K$ exogenous variables as

$$
Y_{w} \Gamma+X B=U
$$

where

$$
Y_{w}=\left(\begin{array}{ccc}
y_{1}(1) & \cdots & y_{M}(1) \\
\vdots & & \vdots \\
y_{1}(T) & & y_{M}(T)
\end{array}\right)
$$

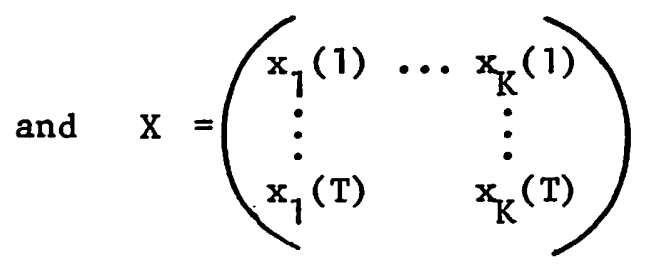

are matrices of observations on $M$ endogenous and $K$ exogenous variables, respectively;

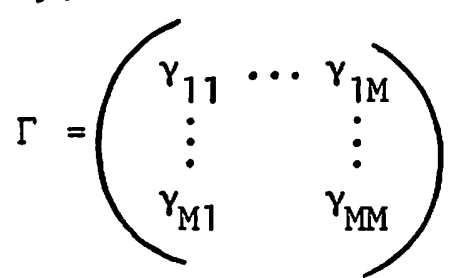
and

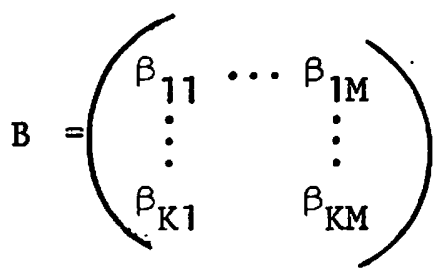

are coefficient matrices, and

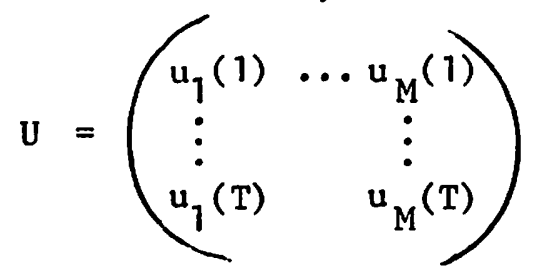

is the $\mathrm{T} \times \mathrm{M}$ matrix of structural disturbances.

We assume that

i) the matrix $\times$ of observations on exogenous variables is purely non-stochastic and fixed in repeated samples, and thus there are no lagged endogenous variables present in the system,

ii) rank $X=K \leq T$, and

$$
\lim _{T \rightarrow \infty}(1 / T) X^{\prime} X=\Sigma_{X X}
$$

is non-singular and positive definite. 
iii) the $\mathrm{T}$ rows of $\mathrm{U}$ are independent random drawings from an M-variate normal population with $\mathrm{EU}=0$ and $(1 / \mathrm{T}) \mathrm{E} \mathrm{U}^{\prime} \mathrm{U}=\Sigma$ where $\Sigma$ is positive definite.

The reduced form of the structural system (2.1) may be written as

$$
\mathrm{Y}_{\mathrm{w}}=\mathrm{X} \Pi_{\mathrm{w}}+\overline{\mathrm{V}}_{\mathrm{w}} \text {, where } \Pi_{\mathrm{w}}=-\mathrm{B} \Gamma^{-1} \text { and } \overline{\mathrm{V}}_{\mathrm{w}}=\mathrm{U} \Gamma^{-1}
$$

provided $\Gamma$ is a non-singular matrix.

It follows that the $T$ rows of $\bar{V}_{w}$ are also $M$-dimensional normal with

$$
E \bar{V}_{w}=0 \text { and }(1 / T) E \bar{V}_{w}^{\prime} \bar{V}_{w}=\Omega=\Gamma^{\prime-1} \Sigma \Gamma^{-1}
$$

where $\Omega$ is then a positive definite matrix.

\section{Estimation of the Reduced Form}

The reduced form coefficients may be estimated either by applying ordinary least squares directly to (2.7), or, by first estimating the structural equations by a consistent method and then deriving the reduced form coefficient estimates. In the former case we have

$$
\hat{\Pi}_{w}=\left(X^{\prime} X\right)^{-1} X^{\prime} Y_{w}
$$

and in the latter

$$
\hat{\Pi}_{w}=-\hat{B} \hat{\Gamma}^{-1} \text {. }
$$

The former procedure may be called unrestricted reduced form estimation, and the latter restricted reduced form estimation. The forecasts obtained according to the two methods have been analyzed by Hooper and Zellner (1961) and Goldberger, Nagar and Odeh (1961), respectively. The comparative merits of the two procedures have been evaluated by several authors. The most detailed evidence comes from the study of Mosbaek and Wold (1969). The main difficulty of the restricted reduced form estimator is that to estimate 
one single reduced form equation one must obtain estimates of coefficients in all structural equations. This is a cumbersome procedure besides being highly sensitive to errors of specification in different structural equations. Amemiya (1966) and Kakwani and Court (1972) have proposed the following method which may be called partially restricted reduced form estimation. Let us consider one single structural equation of the complete system (2.1). After omitting those variables from this equation which enter with zero coefficients and normalizing the coefficients we may write this equation as

$$
y=Y \gamma+X_{1} \beta+u
$$

where $y$ is a $T \times 1$ vector of observations on the left hand endogenous variable, $\mathrm{Y}$ and $\mathrm{X}_{1}$ are $\mathrm{T} \times \mathrm{m}$ and $\mathrm{T} \times \mathrm{K}_{1}$ matrices of observations on the right hand endogenous and exogenous variables, respectively. We have $m+1 \leq M$ and $k_{1} \leq k$. Further, $\gamma$ and $\beta$ are $m \times 1$ and $k_{1} \times 1$ coefficient vectors and $u$ is the $\mathrm{T} \times 1$ vector of structural disturbances.

The reduced form corresponding to $\mathrm{y}$ and $\mathrm{Y}$ may be written as

$$
y=x_{1} \pi^{*}+x_{2} \pi+v=x ? \pi^{*},+v,
$$

and

$$
Y=x_{1} \Pi^{*}+x_{2} \Pi+v=x\left(\begin{array}{l}
\Pi^{*} \\
\Pi
\end{array}\right)+v,
$$

where

$$
x=\left(\begin{array}{ll}
x_{1} & x_{2}
\end{array}\right)
$$

$\mathrm{X}_{2}$ being the $\mathrm{T} \times \mathrm{K}_{2}$ matrix of observations on $\mathrm{K}_{2}$ exogenous variables excluded from (3.3) and $\mathrm{X}_{1}$ is $\mathrm{T} \times \mathrm{K}_{1}$ matrix of $\mathrm{K}_{1}$ exogenous variables included in (3.3); $K=K_{1}+K_{2} \cdot \stackrel{*}{*} \pi$ are reduced form coefficient vectors in (3.4) and $\Pi^{*}, \Pi$ are coefficient matrices in (3.5). The identifiability of (3.3) requires 
(3.7) $\quad \pi^{*}=\Pi^{*} \gamma+\beta$

(3.8) $\pi=\Pi \gamma$

or

(3.9) $\quad\left(\begin{array}{l}\pi^{*} \\ \pi\end{array}\right)=\left(\begin{array}{ll}\Pi^{*} & I \\ \Pi & 0\end{array}\right)\left(\begin{array}{l}\gamma \\ \beta\end{array}\right)$

where $I$ is $K_{1} \times K_{1}$ identity matrix and 0 is null matrix of size $K_{2} \times K_{1}$.

The partially restricted reduced form estimator of the coefficient vector $\left(\begin{array}{l}\pi^{*} \\ \pi\end{array}\right)$, in $(3.4)$, is then defined as

(3.10) $\quad\left(\begin{array}{c}\pi^{*} \\ \hat{\pi}\end{array}\right)=\left(\begin{array}{cc}\left(X^{\prime} X\right)^{-1} X^{\prime} Y & \begin{array}{c}I \\ \end{array} \\ & 0\end{array}\right)\left(\begin{array}{c}c \\ b\end{array}\right)$

where $\left(X^{\prime} X\right)^{-1} X^{\prime} Y$ is the ordinary least squares estimator of $\left(\begin{array}{l}\Pi^{*} \\ \Pi\end{array}\right)$ is (3.5) and

$$
c=\left(Y^{\prime} N Y\right)^{-1} Y^{\prime} N y, \quad b=\left(X_{1}^{\prime} X_{1}\right)^{-1} X_{1}^{\prime}(y-Y c)
$$

are the two-stage least squares estimators of parameters $\gamma$ and $\beta$ in (3.3), respectively;

$$
N=x\left(x^{\prime} x\right)^{-1} x^{\prime}-x_{1}\left(x_{1}^{\prime} x_{1}\right)^{-1} x_{1}^{\prime}
$$

is $\mathrm{T} \times \mathrm{T}$ idempotent symmetric matrix such that

(3.13) $\quad \operatorname{rank} N=\operatorname{tr} N=K-K_{1}=K_{2}$.

It should be noted that the estimator in (3.10) coincides with the unrestricted reduced form estimator in case the structural equation (3.3) is just identified, i.e., $K=m+K_{1}$ :

(3.14) $\quad\left(\begin{array}{l}\tilde{\pi}^{*} \\ \hat{\pi}^{\prime}\end{array}=\left(x^{\prime} \mathrm{x}\right)^{-1} \mathrm{x}^{\prime} \mathrm{y}\right.$ 
4. Analysis of Partially Restricted Reduced Form Forecast Errors in Case (3.3) has only Two Endogenous Variables

If there is only one endogenous variable on the right hand side of

(3.3), i.e., $Y=y_{1}$ is a column vector and $\gamma$ is a scalar coefficient, then we have

(4.1) $\quad y=\gamma y_{1}+x_{1} \beta+u$,

and the reduced form corresponding to $\mathrm{y}$ and $\mathrm{y}_{1}$ may be written as

(4.2) $\quad y=x_{1} \pi^{*}+x_{2} \pi+v=x\left(\begin{array}{l}\pi^{*} \\ \pi\end{array}\right)+v$,

(4.3) $y_{1}=x_{1} \pi_{1}^{*}+x_{2} \pi_{1}+v_{1}=x^{\prime} \pi_{1}^{*}-v_{1} \cdot$

The partially restricted reduced form estimator is

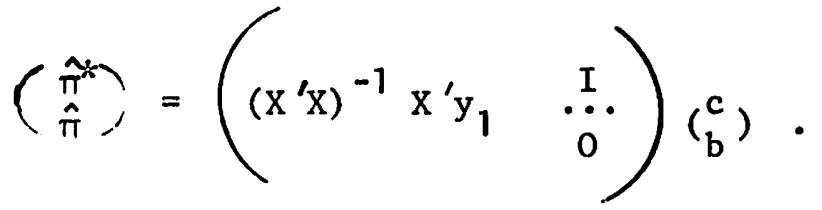

If

$(4.5)$

$$
x_{\tau}^{\prime}=\left(x_{1 \tau} \cdots x_{K \tau}\right)
$$

is the vector of observations, in period of prediction $t=\tau$, on the $K$ exogenous variables, then the forecast value of the left hand endogenous variable in $(4.1)$ is

$$
\hat{y}_{\tau}=x_{\tau}^{\prime}\left(\hat{\pi}^{*}\right)
$$

Since

$$
\text { (4.7) } \quad y_{\tau}=x_{\tau}^{\prime}\left(\pi^{*}+v_{\tau}\right.
$$

according to the reduced form (4.2), and its estimated counterpart is

$$
\text { (4.8) } \quad y_{\tau}=x_{\tau}^{\prime}\left(\begin{array}{l}
\hat{*} \\
\hat{T}
\end{array}+\hat{v}_{\tau}\right. \text {, }
$$

we obtain

$$
\text { (4.9) } \quad \hat{y}_{T}-y_{T}=-\hat{v}_{T}
$$


and

(4.10) $\quad \hat{v}_{\tau}=v_{\tau}-x_{\tau}^{\prime}\left(\begin{array}{ll}\hat{\pi}^{*}-\pi^{*} \\ \hat{\pi}-\pi\end{array}\right)$.

We shall prove the following theorems:

Theorem 1. If the coefficient vector $\left(\begin{array}{l}\pi^{*} \\ \pi\end{array}\right)$ in $(4.2)$ has been estimated according to the partially restricted reduced form procedure indicated in (4.4), then under the Assumptions $i), i i)$ and $i i i)$ stated in Section 2

(4.11) $E \hat{\pi}^{*}=\pi^{*}$ and

(4.12) $\quad E \hat{\pi}=e^{-\theta}\left(\theta f_{12}+\frac{1}{2} f_{01}\right) \pi$

where $\mathrm{K}_{2}>1$ and

(4.13) $\quad \theta=(1 / 2) \bar{y}_{1}^{\prime} \bar{N}_{1}$,

is a certain parameter of non-centrality, $\bar{y}_{1}=E y_{1}$ and $f_{12}, f_{01}$ have been obtained from

(4.14) $\quad \mathrm{f}_{\mathrm{ac}}=\frac{\Gamma\left(\frac{\mathrm{K} 2}{2}+\mathrm{a}\right)}{\Gamma\left(\frac{\mathrm{K}_{2}}{2}+\mathrm{c}\right)} \mathrm{F}_{1}\left(\frac{\mathrm{K}_{2}}{2}+\mathrm{a} ; \frac{\mathrm{K}_{2}}{2}+\mathrm{c} ; \theta\right)$

by specifying $a=1, c=2$ and $a=0, c=1$, respectively; and ${ }_{1} F_{1}()$

is a confluent hypergeometric function.

Using (4.9) and $(4.10)$ we have

(4.15) $\quad E\left(\hat{y}_{T}-y_{\tau}\right)=-E \hat{v}_{T}$

$=x_{\tau}^{\prime}\left(\begin{array}{ll}E \hat{\pi}^{*} & -\pi^{*} \\ E \hat{\pi} & -\pi\end{array}\right)$

and if we partition $x_{T}$ as

(4.16) $\quad x_{T}=\left(\begin{array}{l}x_{*} \\ x_{* * t}\end{array}\right)$

so that $x_{*}$ is the column vector of observations on variables in $x_{1}$ in the 
period $t=T$ of prediction and similarly $x_{* *}$ is the column vector of observations on variables in $x_{2}$ in the period $t=\tau$, then the following result is obtained.

Theorem 2. Under the Assumptions i), ii) and iii) stated in Section 2, the the exact bias of the partially restricted reduced form forecast on the left hand endogenous variable in $(4.1)$ is given by

(4.17) $\quad E\left(\hat{y}_{T}-y_{T}\right)=x_{* *}^{\prime} E(\hat{\pi}-\pi)$

$$
=e^{-\theta}\left(\theta f_{12}+\frac{1}{2} f_{01}-1\right) x_{* *}^{\prime} \pi
$$

where $\mathrm{K}_{2}>1$.

Corollary. For $\theta$ large and positive an asymptotic approximation to the bias of the partially restricted reduced form forecast is given by

$$
\mathrm{E}\left(\hat{\mathrm{y}}_{\tau}-\mathrm{y}_{\tau}\right) \approx \frac{1-\mathrm{K}_{2}}{2} \frac{1}{\theta} \mathrm{x}_{* *}^{\prime} \pi, \mathrm{K}_{2}>1
$$

where terms of lower order than $1 / \theta$ have been omitted.

Theorem 3. Under the Assumptions i), ii) and iii) stated in Section 2, the exact mean squared error of the partially restricted reduced form forecast on the left hand endogenous variable y in (4.1) is given by

$$
E\left(\hat{y}_{T}-y_{T}\right)^{2}=1+x_{*}^{\prime} x_{*}+x_{* *}^{\prime} D x_{* *}
$$

where $x_{\hbar}, x_{t^{\prime}}$ are as defined in $(4.16), K_{2}>2$, and

$$
\begin{aligned}
D=E(\hat{\pi}-\pi)(\hat{\pi}-\pi)^{\prime} & \\
=\left[\left(\frac{1}{2} \theta+\gamma^{2} \theta^{2}\right) I\right. & +\left(1+\frac{K_{2}}{4}+\frac{1}{2} \theta \gamma^{2}\right) \pi_{1} \pi_{1}^{\prime}+\gamma \theta \pi_{1} \pi^{\prime} \\
& \left.+\gamma \theta \pi_{1}^{\prime}\right] e^{-\theta} \mathrm{f}_{13}+\left(\frac{1}{2} \theta+\gamma^{2} \theta^{2}\right) \pi_{1} \pi_{1}^{\prime} e^{-\theta} \mathrm{f}_{24} \\
& +\left[\left(\frac{1}{2}+\frac{K_{2}}{4}+\frac{1}{2} \theta \gamma^{2}\right) I+\frac{1}{2} \pi \pi^{\prime}\right] \mathrm{e}^{-\theta} \mathrm{f}_{02} \\
& -2 \mathrm{e}^{-\theta} \theta \mathrm{f}_{12} \pi \pi^{\prime}-\mathrm{e}^{-\theta} \mathrm{f}_{01} \pi \pi^{\prime}+\pi \pi^{\prime},
\end{aligned}
$$


$f_{13}, f_{24}$ etc. having been obtained from (4.14) by putting $a=1, c=3$;

$a=2, c=4$, etc.

Since, for large and positive value of $\theta$, the asymptotic expansion of the confluent hypergeometric function is given by ${ }^{1}$

$$
\begin{aligned}
& { }_{1} F_{1}\left(\frac{K_{2}}{2}+a ; \frac{K_{2}}{2}+c ; \theta\right) \approx \\
& \frac{\Gamma\left(\frac{K_{2}}{2}+c\right)}{\Gamma\left(\frac{K_{2}}{2}+a\right)} \frac{e^{\theta}}{\theta^{c-a}}\left[1+\frac{(c-a)\left(1-\frac{K_{2}}{2}-a\right)}{1 !} \frac{1}{\theta}+\right. \\
& +\frac{(c-a)(c-a+1)\left(1-\frac{K_{2}}{2}-a\right)\left(1-\frac{K_{2}}{2}-a+1\right)}{2 !} \frac{1}{\theta^{2}} \\
& +\ldots \cdots
\end{aligned}
$$

and $f_{a c}$ is as defined in (4.14) we arrive at the following result.

Corollary. For large values of $\theta$, and $K_{2}>2$, the asymptotic approximation

to the mean squared error: of forecast is given by

$$
\begin{aligned}
E\left(\hat{y}_{\tau}-y_{\tau}\right)^{2} \approx & \\
1 & +x_{*}^{\prime} x_{*}+\gamma^{2} x_{* \star}^{\prime} x_{* *} \\
& +\left[\left(\frac{1+\gamma^{2}}{2}-k_{2} \gamma^{2}\right) x_{* *}^{\prime} x_{* *}+\frac{1-\gamma^{2}}{2} x_{* *}^{\prime} \pi_{1} \pi_{1}^{\prime} x_{* \star}\right] \frac{1}{\theta}
\end{aligned}
$$

where terms of lower order than $\frac{1}{\theta}$ have been neglected.

5. Proof of Theorems 1,2 and 3

The derivation of proof of the theorems stated in the preceding section is considerably simplified if we transform the original structural system (2.1)

${ }^{1} \mathrm{Cf}$. Slater (1950). 
so that the covariance matrix of the reduced form disturbances is unity. Such a transformation of the structural system is always possible and, therefore, there is no loss of generality. Then we assume that $\Omega=I$; and hence

(5.1) $\quad \mathrm{Ev}=\mathrm{Ev} \mathrm{v}_{1}=0$ and

(5.2) $\quad E v v^{\prime}=E v_{1} v_{1}^{\prime}=I$ and $E v v_{1}^{\prime}=0$,

where $I$ is $T \times T$ unit matrix and 0 is $T \times T$ zero matrix.

Further, we assume that

(5.3) $\quad X^{\prime} X=I$.

5.1. Proof of Theorem 1 .

From the equation (4.4) we have

(5.4) $\quad \hat{\pi}^{*}=x_{1}^{\prime} y_{1} c+b$

(5.5) $\quad \hat{\pi}=x_{2}^{\prime} y_{1} c$

where$$
c=\frac{y_{1}^{\prime} \mathrm{Ny}}{y_{1}^{\prime} \mathrm{Ny}_{1}} \text { and } \quad b=x_{1}^{\prime}\left(y-c y_{1}\right) \text {, }
$$

(5.7) $\quad \mathrm{N}=\mathrm{x}_{2} \mathrm{x}_{2}^{\prime}, \mathrm{x}^{\prime} \mathrm{x}=\mathrm{I}$.

Substituting for $b$ in (5.4) from (5.6) we get

(5.8) $\quad \hat{\pi}^{*}=x_{1}^{\prime} y$

and, therefore,

$$
\begin{aligned}
E \hat{\pi}^{*} & =x_{1}^{\prime}(E y)=x_{1}^{\prime}\left(x_{1} \pi^{*}+x_{2} \pi\right) \\
& =\pi^{*}
\end{aligned}
$$

because $x_{1}^{\prime} x_{1}=I$ and $x_{1}^{\prime} x_{2}=0$. 
Let us now consider

$$
\begin{aligned}
\hat{\pi} & =c x_{2}^{\prime} y_{1}=\left(\frac{y_{1}^{\prime} N y_{1}^{\prime}}{y_{1}^{\prime} y_{1}}\right) x_{2}^{\prime} y_{1} \\
& =\left(\frac{y_{1}^{\prime} x_{2} x_{2}^{\prime} y}{y_{1}^{\prime} x_{2} x_{2}^{\prime} y_{1}}\right) x_{2}^{\prime} y_{1}=\left(\frac{z_{1}^{\prime} z^{\prime}}{z_{1}^{\prime} z_{1}}\right) z_{1}
\end{aligned}
$$

where we write

$$
z=x_{2}^{\prime} y \text { and } \quad z_{1}=x_{2}^{\prime} y_{1}
$$

It should be noted that the elements of $z$ and $z_{1}$ are independently normally distributed with

$$
\left\{\begin{array}{l}
\bar{z}=E z=X_{2}^{\prime}(E y)=X_{2}^{\prime} \bar{y}=x_{2}^{\prime}\left(x_{1} \pi^{*}+x_{2} \pi\right)=\pi \\
\bar{z}_{1}=E z_{1}=X_{2}^{\prime}\left(E y_{1}\right)=x_{2}^{\prime} \bar{y}_{1}=x_{2}^{\prime}\left(x_{1} \pi_{1}^{*}+x_{2} \pi_{1}\right)=\pi_{1}
\end{array}\right.
$$

and

$$
\left\{\begin{array}{l}
E(z-\bar{z})(z-\bar{z})^{\prime}=E\left(z_{1}-\bar{z}_{1}\right)\left(z_{1}-\bar{z}_{1}\right)^{\prime}=I \text { and } \\
E(z-\bar{z})\left(z_{1}-\bar{z}_{1}\right)^{\prime}=0,
\end{array}\right.
$$

I being $\mathrm{K}_{2} \times \mathrm{K}_{2}$ unit matrix and 0 is $\mathrm{K}_{2} \times \mathrm{K}_{2}$ zero matrix.

If we write $z_{1 i}$ and $z_{i}$ for the $i^{\text {th }}$ elements of $z_{1}$ and $z$, respectively, then the $\mathrm{j}^{\text {th }}$ element of the vector in (5.10) is given by

$$
\hat{\pi}_{j}=\left(\frac{\sum_{i=1}^{K_{2}} z_{1 i} z_{i}}{K_{i=1} z_{1 i}^{2}}\right) z_{1 j}
$$

Hence

$$
E \hat{\pi}_{j}=E\left(\frac{z_{1 j}^{2}}{\sum_{i=1}^{K_{2}} z_{1 i}^{2}}\right) E z_{j}+\sum_{i \neq j=1}^{K_{2}} E\left(\frac{z_{1 i} z_{1 j}}{K_{2}}\right) E z_{i},
$$


and using results obtained in (A.4) and (A.5) of the appendix we arrive at the result stated in $(4.12)$.

\subsection{Proof of Theorem 2}

The proof- of the Theorem 2 has already been outlined in the text preceding the statement of the theorem. Further, the result in corollary to the Theorem 2 follows in a straight forward manner by using asymptotic expansion of the relevant confluent hypergeometric functions.

\subsection{Proof of Theorem 3}

Using (4.9) and (4.10) the mean squared error of forecast on the left hand endogenous variable in $(4.1)$ is given by

$(5.16) E\left(\hat{y}_{\tau}-y_{T}\right)^{2}=E \hat{v}_{T}^{2}$

$$
=E\left[v_{\tau}-x_{\tau}^{\prime}\left(\begin{array}{c}
\hat{\pi}^{*}-\pi^{*} \\
\hat{\pi}-\pi
\end{array}\right)\right], r_{-}^{r} v_{\tau}-x_{\tau}^{\prime}\left(\begin{array}{l}
\hat{\pi}^{*}-\pi^{*} \\
\hat{\pi}-\pi
\end{array}\right) \cdot
$$

Since

$$
\text { (5.17) } \quad x_{\tau}=\left(\begin{array}{l}
x_{*} \\
x_{t * *}
\end{array}\right)
$$

according to $(4.16)$, we have

$$
\begin{aligned}
E\left(\hat{y}_{\tau}-y_{T}\right)^{2} & =1+x_{*}^{\prime} E\left(\hat{\pi}^{*}-\pi^{*}\right)\left(\hat{\pi}^{*}-\pi^{*}\right)^{\prime} x_{*} \\
& +2 x_{*}^{\prime} E\left(\hat{\pi}^{*}-\pi^{*}\right)(\hat{\pi}-\pi)^{\prime} x_{k *} \\
& +x_{* *}^{\prime} E(\hat{\pi}-\pi)(\hat{\pi}-\pi)^{\prime} x_{* \star}
\end{aligned}
$$

because due to the assumption of temporal independence of disturbances and zero mean of disturbances other terms on the right hand side vanish, and $\mathrm{Ev}_{\tau}^{2}=1$ 
Let us now observe that

$$
E\left(\hat{\pi}^{*}-\pi^{*}\right)\left(\hat{\pi}^{*}-\pi^{*}\right)^{\prime}=\mathrm{E} \hat{\pi}^{*} \hat{\pi}^{* \prime}-\pi^{*} \pi^{* \prime}
$$

because $E \hat{\pi}^{*}=\pi^{*}$ as shown in (4.11), and using (5.8) we have

$$
\begin{aligned}
\mathrm{E} \hat{\pi}^{*} \hat{\pi}^{*} & =E \mathrm{x}_{1}^{\prime} \mathrm{yy}^{\prime} \mathrm{x}_{1}=\mathrm{x}_{1}^{\prime}\left(\mathrm{E} \text { y }{ }^{\prime}\right) \mathrm{x}_{1} \\
& =\mathrm{x}_{1}^{\prime}\left(I+\overline{\mathrm{y}} \bar{y}^{\prime}\right) \mathrm{x}_{1}=I+\pi^{*} \pi^{*}
\end{aligned}
$$

because $E(y-\bar{y})(y-\bar{y})^{\prime}=I, \bar{y}=E y$ and $x^{\prime} X=I$. Therefore,

(5.21) $\quad E\left(\hat{\Pi}^{*}-\pi^{*}\right)\left(\hat{\pi}^{*}-\pi^{*}\right)^{\prime}=I$,

and

(5.22) $\quad x_{*}^{\prime} E\left(\hat{\pi}^{*}-\pi^{*}\right)\left(\hat{\pi}^{*}-\pi^{*}\right)^{\prime} x_{*}=x_{*}^{\prime} x_{*}$

Next let us consider

$$
E\left(\hat{\pi}^{*}-\pi^{*}\right)(\hat{\pi}-\pi)^{\prime}=E \hat{\pi}^{*} \hat{\Pi}^{\prime}-\pi^{*}\left(E \hat{\pi}^{\prime}\right),
$$

and note that

(5.24) $\hat{\pi}^{*}=x_{1}^{\prime} y$ and $\hat{\pi}=c x_{2}^{\prime} y_{1}$

from (5.8) and (5.5), respectively; and

(5.25) $\quad c=\frac{y_{1}^{\prime} x_{2} x_{2}^{\prime} y}{y_{1}^{\prime} x_{2} x_{2}^{\prime} y_{1}}$.

Therefore, $\hat{\pi}^{*}$ is independently distributed of $\hat{\pi}$, as $x_{1}^{\prime} X_{2}=0$. Hence (5.26) $\mathrm{E} \hat{\pi}^{*} \hat{\pi}^{\prime}=\mathrm{E} \hat{\pi} * \mathrm{E} \hat{\pi}^{\prime}=\pi^{*} \mathrm{E} \hat{\pi}^{\prime}$

because $E \hat{\pi}^{*}=\pi^{*}$, and

$$
E\left(\hat{\Pi}^{*}-\pi^{*}\right)(\hat{\pi}-\pi)^{\prime}=0 \text {. }
$$

Finally, let us obtain the value of

$$
E(\hat{\Pi}-\pi)(\hat{\leftarrow}-\pi)^{\prime}=E \hat{\pi} \hat{\pi}^{\prime}-(E \hat{\pi}) \pi^{\prime}-\pi(E \hat{\pi})^{\prime}+\pi \pi^{\prime}
$$

where $E \hat{\Pi}$ is already given in $(4.12)$. We can write 
(5.29) $\begin{aligned} \hat{\pi} \hat{\pi}^{\prime} & =c^{2} x_{2}^{\prime} y_{1} y_{1}^{\prime} x_{2}=\left(\frac{y_{1}^{\prime} x_{2} x_{2}^{\prime} y}{y_{1}^{\prime} x_{2} x_{2}^{\prime} y_{1}}\right)^{2} x_{2}^{\prime} y_{1} y_{1}^{\prime} x_{2} \\ & =\left(\frac{z_{1}^{\prime} z}{z_{1}^{\prime} z_{1}}\right) z_{1} z_{1}^{\prime}\end{aligned}$

where $z_{1}=x_{2}^{\prime} y_{1}$ and $z=x_{2}^{\prime} y$.

If we write $z_{1 i}, z_{i} \quad\left(i=1, \ldots, k_{2}\right)$ for the $i^{\text {th }}$ elements of $z_{1}$ and $z$, respectively; then the diagonal element in the $k^{\text {th }}$ row and $k^{\text {th }}$ column of $\hat{\pi} \hat{\pi}^{\prime}$ is

$(5.30)$

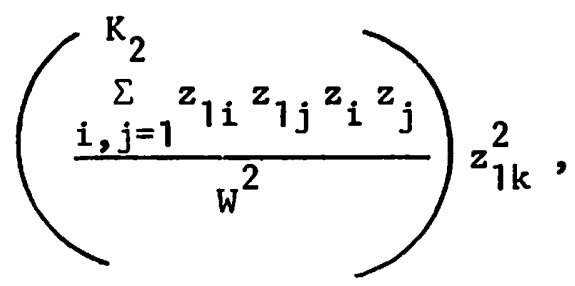

and the off-diagonal element in the $k^{\text {th }}$ row and the $l^{\text {th }}$ column is

where

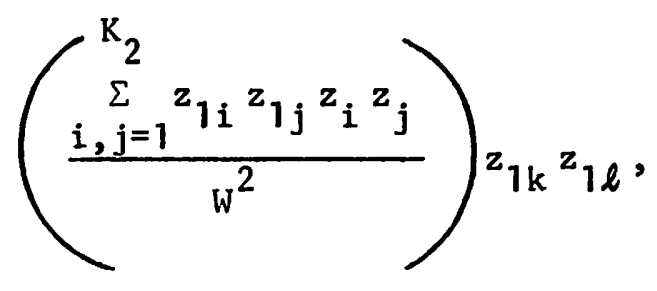

$$
\text { (5.32) } \quad W=\sum_{i=1}^{K} z_{1 i}^{2}
$$

is a non-central $x^{2}$ variate with $K_{2}$ degrees of freedom and

(5.33) $\quad \theta=\frac{1}{2} \sum_{i=1}^{K_{2}} \bar{z}_{1 i}^{-2}$

is the parameter of non-centrality, $\bar{z}_{1 i}=E z_{1 i}$.

It can be verified easily that

(5.34) $\frac{1}{2} \sum_{i=1}^{K_{2}} \bar{z}_{i}^{-2}=\theta \gamma^{2}$, and $\frac{1}{2} \sum_{i=1}^{K_{2}} \bar{z}_{i} \bar{z}_{1 i}=\gamma \theta$. 
Let us now evaluate the mathematical expectation of the diagonal element (5.30) in the first instance.

We will distinguish five cases as below:

\begin{tabular}{|c|c|}
\hline Cases & Mathematical Expectation of (5.30) \\
\hline$i=j=k$ & $E\left(z_{1 k}^{4} / w^{2}\right) E z_{k}^{2}$ \\
\hline$i=j$ but $\neq k$ & $\sum_{\substack{i=1 \\
\neq k}}^{K} E\left(z_{1 i}^{2} z_{1 k}^{2} / W^{2}\right) E z_{i}^{2}$ \\
\hline$i=k$ but $\neq j$ & $\sum_{\substack{j=1 \\
\neq k}}^{K} E\left(z_{1 k}^{3} z_{1 j} / W^{2}\right) E z_{k} E z_{j}$ \\
\hline $\mathrm{j}=\mathrm{k} \quad$ but $\neq \mathrm{i}$ & $\sum_{\substack{i=1 \\
\neq k}}^{K_{2}} E\left(z_{1 i} z_{1 k}^{3} / W^{2}\right) E z_{i} E z_{k}$ \\
\hline$i \neq j \neq k$ & $\sum_{\substack{i, j=1 \\
i \neq j \neq k}}^{K_{2}} E\left(z_{1 i} z_{1 j} z_{1 k}^{2} / W^{2}\right) E z_{i} E z_{j}$ \\
\hline
\end{tabular}

Mathematical expectations required above are given in the Appendix (A.6) to (A.9); and we note that

(5.35) $E z_{i}=\bar{z}_{i}$ and $V z_{i}=E z_{i}^{2}-\left(E z_{i}\right)^{2}=1$ or $E z_{i}^{2}=1+z_{i}^{2}$

Therefore, the mathematical expectation of the diagonal term given in $(5.30)$ is 
16

$(5.36)$

$$
\begin{aligned}
& \sum_{i, j=1}^{k_{2}} E\left(\frac{z_{1 i^{z} 1 j_{1 k}}^{2}}{W^{2}}\right) E z_{i} z_{j}= \\
& {\left[\left(\frac{1}{2} \theta+\gamma^{2} \theta^{2}\right)+\left(1+\frac{K_{2}}{4}+\frac{1}{2} \theta \gamma^{2}\right) \bar{z}_{1 k}^{-2}+2 \gamma \theta \bar{z}_{1 k} \bar{z}_{k}\right] e^{-\theta} f_{13}} \\
& +\left[\frac{1}{2} \theta+\gamma^{2} \theta^{2}\right] \bar{z}_{1 k}^{2} e^{-\theta} f_{24} \\
& +\left[\left(\frac{1}{2}+\frac{K_{2}}{4}+\frac{1}{2} \theta \gamma^{2}\right)+\frac{1}{2} z_{k}^{2}\right] e^{-\theta} f_{02} .
\end{aligned}
$$

Next let us consider the mathematical expectation of the off-diagonal element given in (5.31).

We will distinguish the following cases:

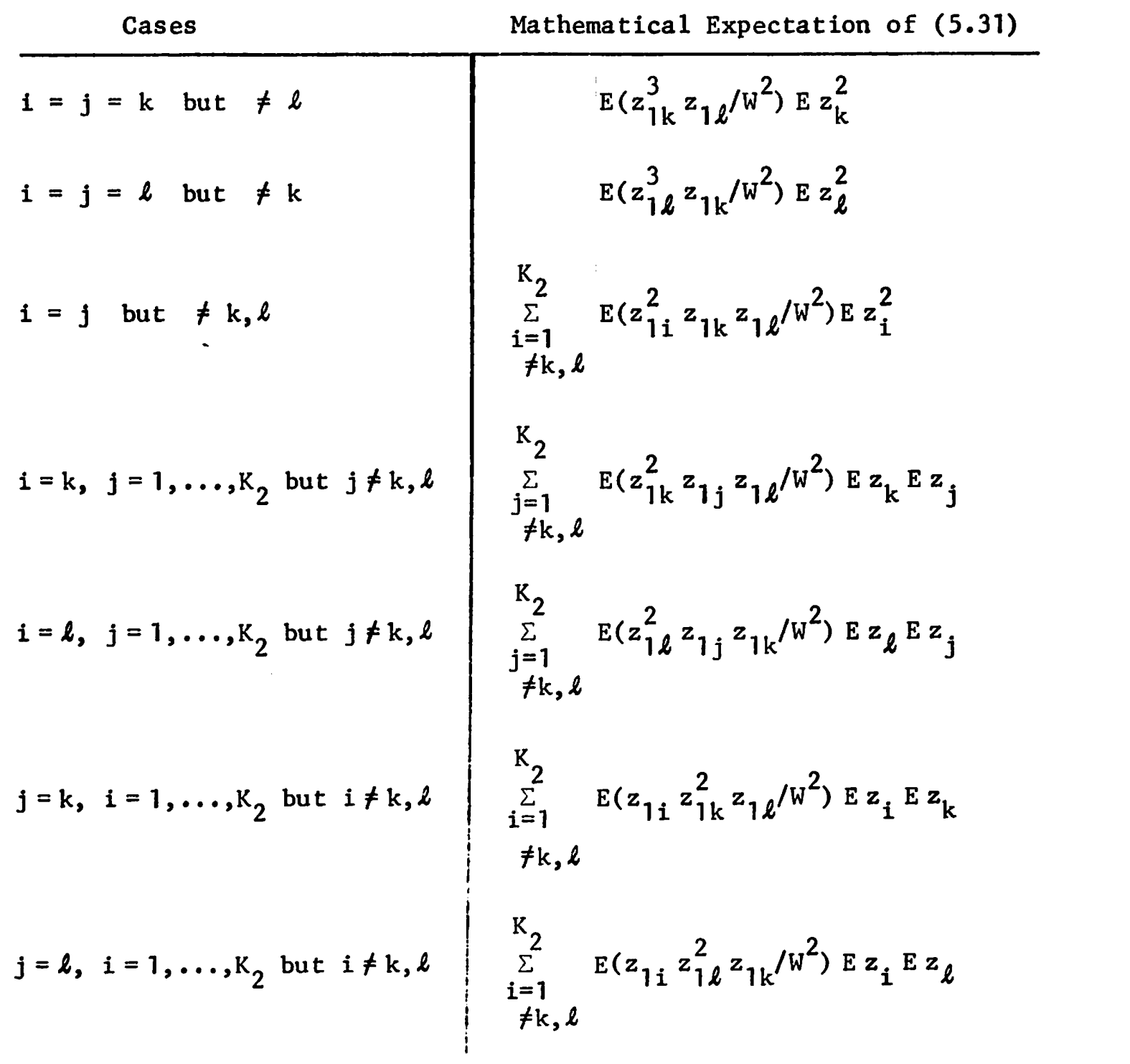




\begin{tabular}{l|c} 
Cases & Mathematical Expectation of (5.31) \\
\hline$i=k, j=\ell$ but $i \neq j$ & $E\left(z_{1 k}^{2} z_{1 \ell}^{2} / w^{2}\right) E z_{k} E z_{\ell}$ \\
$i \neq j \neq k \neq l$ & $E\left(z_{1 \ell}^{2} z_{1 k}^{2} / w^{2}\right) E z_{\ell} E z_{k}$ \\
$\sum_{\substack{i, j=1 \\
i \neq j \neq k \neq l}}^{k_{2}} E\left(z_{1 i} z_{1 j} z_{1 k} z_{1 \ell} / W^{2}\right) E z_{i} E z_{j}$
\end{tabular}

The mathematical expectations required in the above table are given in the Appendix (A.7) to (A.10).

Therefore, the mathematical expectation of the term given in (5.31)

is

$$
\begin{aligned}
{\left[\left(1+\frac{\mathrm{K}_{2}}{4}+\frac{1}{2} \theta \gamma^{2}\right) \bar{z}_{1 \mathrm{k}} \bar{z}_{1 \ell}\right.} & \left.+\gamma \theta \bar{z}_{1 \ell} \bar{z}_{k}+\gamma \theta \bar{z}_{1 k} \bar{z}_{\ell}\right] \mathrm{e}^{-\theta} \mathrm{f}_{13} \\
& +\left(\frac{1}{2} \theta+\gamma^{2} \theta^{2}\right) \bar{z}_{1 k} \bar{z}_{1 \ell} \mathrm{e}^{-\theta} \mathrm{f}_{24} \\
& +\frac{1}{2} \bar{z}_{k} \bar{z}_{\ell} e^{-\theta} \mathrm{f}_{02} .
\end{aligned}
$$

Therefore, using (5.29), we have

$$
\begin{aligned}
E \hat{\pi} \hat{\pi}^{\prime}=\left[\left(\frac{1}{2} \theta+\gamma^{2} \theta^{2}\right) I\right. & +\left(1+\frac{K_{2}}{4}+\frac{1}{2} \theta \gamma^{2}\right) \bar{z}_{1} \bar{z}_{1}^{\prime}+\gamma \theta_{z_{1}} \bar{z}^{\prime}+ \\
& \left.+\gamma \theta \bar{z} \bar{z}_{1}^{\prime}\right] e^{-\theta} f_{13} \\
& +\left(\frac{1}{2} \theta+\gamma^{2} \theta^{2}\right) \bar{z}_{1} \bar{z}_{1}^{\prime} e^{-\theta} \mathrm{f}_{24} \\
& +\left[\left(\frac{1}{2}+\frac{K_{2}}{4}+\frac{1}{2} \theta \gamma^{2}\right) I+\frac{1}{2} \bar{z} \bar{z}^{\prime}\right] e^{-\theta} \mathrm{f}_{02} ;
\end{aligned}
$$

and finally using $(5.28)$ we get 


$$
\text { (5.39) } \begin{aligned}
E(\hat{\pi}-\pi)(\hat{\pi}-\pi)^{\prime} & =\left[\left(\frac{1}{2} \theta+\gamma^{2} \theta^{2}\right) I\right. \\
& \left.+\left(1+\frac{K_{2}}{4}+\frac{1}{2} \theta \gamma^{2}\right) \bar{z}_{1} \bar{z}_{1}^{\prime}+\gamma \theta_{\bar{z}_{1}} \bar{z}^{\prime}+\gamma \theta \bar{z} \bar{z}_{1}^{\prime}\right] e^{-\theta} f_{13} \\
& +\left(\frac{1}{2} \theta+\gamma^{2} \theta^{2}\right) \bar{z}_{1} \bar{z}_{1}^{\prime} e^{-\theta} f_{24} \\
& +\left[\left(\frac{1}{2}+\frac{k_{2}}{4}+\frac{1}{2} \theta \gamma^{2}\right) I+\frac{1}{2} \bar{z} \bar{z}^{\prime}\right] e^{-\theta} f_{02} \\
& -2 e^{-\theta} \theta f_{12} \pi \pi^{\prime}-e^{-\theta} f_{01} \pi \pi^{\prime}+\pi \pi^{\prime} .
\end{aligned}
$$

It should be noted that

$$
\bar{z}=E z=E\left(X_{2}^{\prime} y\right)=\pi \text { and } \quad \bar{z}_{1}=E z_{1}=E\left(X_{2}^{\prime} y_{1}\right)=\pi_{1} \text { because } X^{\prime} X=I \text {. }
$$




\section{$\underline{\text { References }}$}

Amemiya, T (1966): "On the Use of Principal Components of Independent Variables in Two-Stage Least-Squares Estimation."

International Economic Review, 7, pp. 283-303.

Goldberger, A. S., A. L. Nagar, and H. S. Odeh (1961): "The Covariance Matrices of Reduced-Form Coefficients and of Forecasts for a Structural Econometric Mode1." Econometrica, 29, pp. 556-573.

Hooper, J. W., and A. Zellner (1961): "The Error of Forecasts for Multivariate Regression Mode1s." Econometrica, 29, pp. 544-555.

Kakwani, N. C., and R. H. Court (1972): "Reduced-Form Coefficient Estimation and Forecasting from a Simultaneous Equation Model." Australian Journal of Statistics, 14, pp. 143-160.

Mosbaek, E. J., and H. O. Wold (1969): Interdependent Systems-Structure and Estimation. North-Holland.

Nagar, A. L., and Aman U11ah (1973): "Note On Approximate Skewness and Kurtosis of the Two-Stage Least-Squares Estimator." Indian Economic Review, 8, pp. 69-80.

Nagar, A. L., and S. N. Sahay (1974): "Exact Mean of the Two-Stage LeastSquares Residuals in a Structural Equation With Two Endogenous Variables." Working Paper No. 22, Australian National University.

Rao, C. R. (1965): Linear Statistical Inference and Its Applications. John Wiley and Son, New York.

Slater, L. J. (1960): Confluent Hypergeometric Functions, Cambridge University Press, Cambridge. 


\section{Appendix}

If $z_{11}, \ldots, z_{1 K_{2}}$ are independently normal with

(A.1) $\quad E z_{1 i}=\bar{z}_{1 i}$ and $\quad V z_{1 i}=1, i=1, \ldots, K_{2}$

then

(A.2) $\quad W=\sum_{i=1}^{K_{2}} z_{1 i}^{2}$

is distributed according to Non-Central Chi-Square distribution with $\mathrm{K}_{2}$ degrees of freedom and

(A.3) $\quad \theta=\frac{1}{2} \sum_{i=1}^{K_{2}} z_{1 i}^{-2}$

is the parameter of non-centrality.

The following mathematical expectations have been worked out by Nagar and Aman Ullah (1973):

(A.4) $\quad E\left(z_{1 i}^{2} W^{-1}\right)=\frac{1}{2} e^{-\theta}\left[\bar{z}_{1 i}^{2} f_{12}+f_{01}\right]$

(A.5) $\quad E\left(z_{1 i} z_{1 j} W^{-1}\right)=\frac{1}{2} \bar{z}_{1 i} \bar{z}_{1 j} e^{-\theta} f_{12}, \quad i \neq j$

where $f_{12}, f_{01}$ have been obtained from (4.14) by writing $a=1, c=2$ and $\mathrm{a}=0, \mathrm{c}=1$, respectively.

Further,

(A.6) $\quad E\left(z_{1 i}^{4} W^{-2}\right)=\frac{1}{4} z_{1 i}^{-4} e^{-\theta} f_{24}+\frac{6}{4} z_{1 i}^{-2} e^{-\theta} f_{13}+\frac{3}{4} e^{-\theta} f_{02}$

(A.7) $\quad E\left(z_{1 i}^{2} z_{1 j}^{2} W^{-2}\right)=\frac{1}{4} z_{1 i}^{-2} z_{1 j}^{-2} e^{-\theta} f_{24}+\frac{1}{4}\left(z_{1 i}^{-2}+z_{1 j}^{-2}\right) e^{-\theta} f_{13}+\frac{1}{4} e^{-\theta} f_{02}, i \neq j$

(A.8) $\quad E\left(z_{1 i}^{3} z_{1 j} W^{-2}\right)=\frac{1}{4} \bar{z}_{1 i}^{-3} \bar{z}_{1 j} e^{-\theta} f_{24}+\frac{3}{4} \bar{z}_{1 i} \bar{z}_{1 j} e^{-\theta} f_{13}, \quad i \neq j$

(A.9) $\quad E\left(z_{1 i} z_{1 j} z_{1 k}^{2} W^{-2}\right)=\frac{1}{4} \bar{z}_{1 i} \bar{z}_{1 j} \bar{z}_{1 k} e^{-\theta} f_{24}+\frac{1}{4} \bar{z}_{1 i} \bar{z}_{1 j} e^{-\theta} f_{13}, \quad i \neq j \neq k$ 


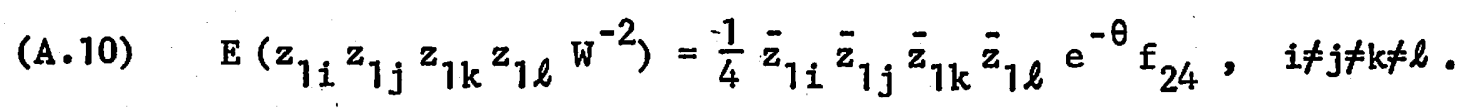

